\title{
Self-Assembled Lecithin/Chitosan Nanoparticles Based on Phospholipid Complex: A Feasible Strategy to Improve Entrapment Efficiency and Transdermal Delivery of Poorly Lipophilic Drug
}

This article was published in the following Dove Press journal:

International Journal of Nanomedicine

\author{
Wujun Dong ${ }^{1,2, *}$ \\ Jun $\mathrm{Ye}^{1,2, *}$ \\ Weijue Wang ${ }^{1,2}$ \\ Yanfang Yang ${ }^{1,2}$ \\ Hongliang Wang ${ }^{1,2}$ \\ Tong Sun ${ }^{1,2}$ \\ Lili Gao ${ }^{1,2}$ \\ Yuling Liu ${ }^{1,2}$ \\ 'State Key Laboratory of Bioactive \\ Substance and Function of Natural \\ Medicines, Institute of Materia Medica, \\ Chinese Academy of Medical Sciences \& \\ Peking Union Medical College, Beijing \\ I00050, People's Republic of China; \\ ${ }^{2}$ Beijing Key Laboratory of Drug Delivery \\ Technology and Novel Formulation, \\ Institute of Materia Medica, Chinese \\ Academy of Medical Sciences \& Peking \\ Union Medical College, Beijing 100050, \\ People's Republic of China
}

*These authors contributed equally to this work

\begin{abstract}
Purpose: Lecithin/chitosan nanoparticles have shown great promise in the transdermal delivery of therapeutic agents. Baicalein, a natural bioactive flavonoid, possesses multiple biological activities against dermatosis. However, its topical application is limited due to its inherently poor hydrophilicity and lipophilicity. In this study, the baicalein-phospholipid complex was prepared to enhance the lipophilicity of baicalein and then lecithin/chitosan nanoparticles loaded with the baicalein-phospholipid complex were developed to improve the transdermal retention and permeability of baicalein.
\end{abstract}

Methods: Lecithin/chitosan nanoparticles were prepared by the solvent-injection method and characterized in terms of particle size distribution, zeta potential, and morphology. The in vitro release, the ex vivo and in vivo permeation studies, and safety evaluation of lecithin/ chitosan nanoparticles were performed to evaluate the effectiveness in enhancing transdermal retention and permeability of baicalein.

Results: The lecithin/chitosan nanoparticles obtained by the self-assembled interaction of chitosan and lecithin not only efficiently encapsulated the drug with high entrapment efficiency $(84.5 \%)$ but also provided sustained release of baicalein without initial burst release. Importantly, analysis of the permeation profile ex vivo and in vivo demonstrated that lecithin/chitosan nanoparticles prolonged the retention of baicalein in the skin and efficiently penetrated the barrier of stratum corneum without displaying skin irritation.

Conclusion: These results indicate the potential of drug-phospholipid complexes in enhancing the entrapment efficiency and self-assembled lecithin/chitosan nanoparticles based on phospholipid complexes in the design of a rational transdermal delivery platform to improve the efficiency of transdermal therapy by enhancing its percutaneous retention and penetration in the skin.

Keywords: lecithin/chitosan nanoparticles, transdermal delivery, phospholipid complex, baicalein

\section{Introduction}

Self-assembled lecithin/chitosan nanoparticles (LCNs) have attracted extensive attention as tools for effective diagnosis, drug delivery, and therapy monitoring. This is partly because LCNs possess advantages such as simple and mild self-assembled process, suitable biocompatibility, and biodegradability. ${ }^{1-3}$ The effectiveness of LCNs is as a result of the desirable characteristics of the constitutive materials. Chitosan, a natural positively charged polysaccharide, is a frequently applied mucoadhesive polymer with
State Key Laboratory of Bioactive Substance and Function of Natural Medicines, Institute of Materia Medica, Chinese Academy of Medical Sciences \& Peking Union Medical College, Beijing 100050, People's Republic of China

Tel +86 1089285188

Fax +86 1089285190

Email ylliu@imm.ac.cn

International Journal of Nanomedicine 2020:I5 5629-5643 
various unique biological characteristics, such as remarkable bioadhesive to mucosal surfaces and ability to open tight junctions in epithelial cells to strongly promote drug penetration. $^{3-7}$ Previous studies have demonstrated the great potential of therapeutic agents-encapsulated nano- and microparticles as well as hydrogels-based chitosan for the treatment of various diseases, such as inflammation, leprosy, and certain cancer-related diseases. ${ }^{8-10}$ Lecithin, a negatively charged lipid mixture of phospholipids, is the primary constituent of cell membranes and has been commonly used for the preparation of various nanoparticles. ${ }^{11-14}$ LCNs are formed through self-assembly of negatively charged lecithin and positively charged chitosan via electrostatic interactions. The inner core is the hydrophobic lipid and can be utilized for encapsulation of lipophilic drugs, whereas the hydrophilic chitosan constitutes the outer shell and provides stabilization. ${ }^{1}$ LCNs possess the integrated advantages of lecithin and chitosan, which can extend the retention time of drugs at the target site and promote drug penetration. ${ }^{1-3}$ Due to the enhanced mucoadhesiveness and penetration, LCNs hold great potential to improve the permeation of encapsulated drugs across various biological barriers by transdermal, mucosal, ocular and oral routes. ${ }^{1,3,4,6,15,16}$

Drugs are encapsulated with LCNs for effective delivery and penetration at the site of action. The lipophilicity of drugs plays a key role in entrapment efficiency. ${ }^{17}$ Previous studies report that lipophilic compounds, such as curcumin, progesterone, diflucortolone, and clobetasol propionate, which commonly distribute in the hydrophobic lipid core by physical embedding or electrostatic interactions with phospholipid, can be encapsulated effectively into LCNs. $^{16-18}$ However, the encapsulation of poorly lipophilic compounds, such as melatonin and metoclopramide, is challenging and leads to extremely low entrapment efficiency and relatively poor stability. ${ }^{17,19}$ Two typical strategies have been proposed to improve the lipid solubility of drugs: modification of chemical structure and preparation of drug-phospholipid complexes. ${ }^{20,21}$ Drug-phospholipid complexes are formed by non-covalent interactions between active compounds and the polar head of phospholipids. ${ }^{21}$ Drug-phospholipid complexes are preferred over modification of chemical structure, as they are biodegradable and non-toxic, thus they are used as lipophilic intermediates for encapsulation into nanocarriers. ${ }^{22}$

Baicalein, a natural bioactive flavonoid isolated from the traditional Chinese herbal medicine huangqin, has been reported to exhibit multiple biological activities, especially anti-inflammatory and anti-bacterial, making it a promising candidate for transdermal therapy of dermatosis, including ultraviolet-induced skin damage. ${ }^{23,24}$ However, the transdermal delivery of baicalein is restricted due to its poor hydrophilicity and lipophilicity. ${ }^{25}$ As LCNs have been reported to exhibit many potential advantages in terms of bioadhesives and penetration, encapsulation of baicalein in LCNs may enhance drug retention and facilitate drug penetration through the skin barrier. However, baicalein is a poorly hydrophilic and lipophilic compound that cannot be loaded effectively into nanocarriers. ${ }^{26,27}$ To enhance the entrapment efficiency and stability, baicalein-phospholipid complex can be prepared to improve the lipid solubility of baicalein and used as a lipophilic intermediate. ${ }^{26,28}$

In this study, LCNs loaded with baicalein-phospholipid complex (BPC-LCNs) were developed to improve the transdermal retention and permeability of baicalein (Scheme 1). BPC-LCNs were prepared by the solvent-injection method and characterized in terms of particle size distribution, zeta potential, and morphology. Moreover, the in vitro release, the ex vivo and in vivo permeation studies of BPC-LCNs were performed to evaluate effectiveness in enhancing transdermal retention and permeability of baicalein. In addition, the histopathology examination of skin was carried out to investigate the mechanism of enhanced permeation and the skin irritation test was conducted to evaluate the safety of the topical application.

\section{Materials and Methods}

\section{Materials}

Baicalein (purity 98\%) was provided by Jiangsu Zelang Medical Technology Co., Ltd. (Nanjing, China). Chitosan, with the viscosity of 20-300 cP and the degree of deacetylation of $75-85 \%$, was purchased from Sigma-Aldrich (St. Louis, MO, USA). Soybean phospholipids (Lipoid S75) were purchased from Lipoid GmbH (Ludwigshafen, Germany). All other reagents, including Tween- 80 and ethanol, were of analytical grade and were purchased from Sinopharm Chemical Reagent Co., Ltd. (Shanghai, China).

\section{Preparation of Baicalein-Phospholipid Complex (BPC)}

BPC was prepared using a solvent evaporation method. A moderate amount of baicalein and phospholipids (1:3.5, w/w) was accurately weighed (MS104S; Mettler-Toledo $\mathrm{GmbH}$, Zurich, Switzerland) and dissolved in tetrahydrofuran with magnetic stirring. After complete dissolution, the organic solvent was evaporated in a rotary evaporator 


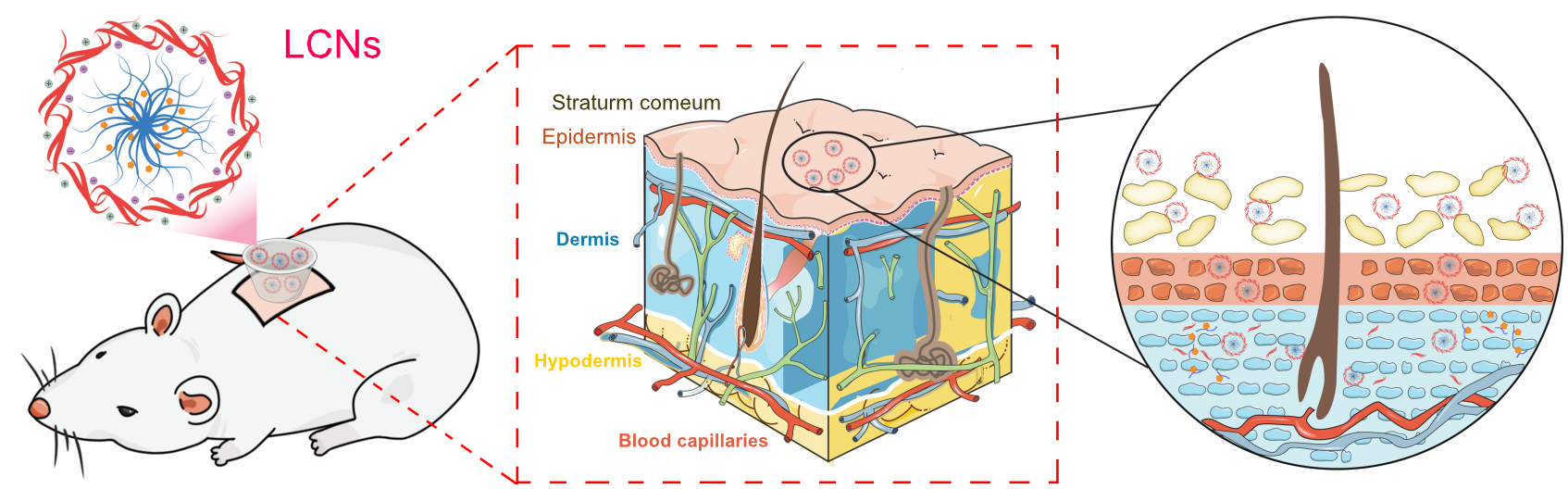

Scheme I Schematic overview of BPC-LCNs that improves the retention of baicalein in the skin and further penetrates the barrier of stratum corneum. Abbreviation: BPC-LCNs, lecithin/chitosan nanoparticles loaded with baicalein-phospholipid complex.

under reduced pressure to obtain a solid residue. The resulting BPC was gently scraped off and stored in a desiccator at room temperature.

\section{Preparation and Characterization of Nanoparticles}

Lecithin/chitosan nanoparticles (LCNs) loaded with baicalein-phospholipid complex (BPC), abbreviated as BPCLCNs, were prepared following the solvent-injection method reported previously. ${ }^{29}$ Chitosan $(10 \mathrm{mg} / \mathrm{mL})$ was weighed and dissolved in $1 \%(\mathrm{v} / \mathrm{v})$ acetic acid overnight at $4^{\circ} \mathrm{C}$. Further, chitosan solution was prepared by adding the aliquots of the above solution to $4 \%(\mathrm{w} / \mathrm{v})$ Tween-80. BPC was dissolved in ethanol $(25 \mathrm{mg} / \mathrm{mL})$ and then added to the ethanolic solution of $2.5 \%(\mathrm{w} / \mathrm{v})$ lecithin. BPC-LCNs were obtained by injecting BPC dissolved in lecithin solution into the chitosan solution with continuous mild stirring for $30 \mathrm{~min}$ at room temperature. For comparison, lecithin/chitosan nanoparticles (LCNs) loaded with baicalein (BA), abbreviated as BA-LCNs, were prepared using the same procedure, except that baicalein was dissolved in ethanol. Ethosomes loaded with baicalein-phospholipid complex (BPC), abbreviated as BPC-ETHs, were prepared following the injection-ultrasonic method. BPC and lecithin were dissolved in ethanol and then added to $1.25 \%(\mathrm{w} / \mathrm{v})$ Tween-80 with continuous moderate stirring for $5 \mathrm{~min}$. BPC-ETHs were then, obtained by sonication for $8 \mathrm{~min}$.

Nanoparticles were analyzed for mean particle size, size distribution (polydispersity index, PdI), and zeta potentials by the dynamic light scattering (DLS) method using the Zeta Potential/Particle Sizer NICOMP 380 ZLS (PSS NICOMP, Santa Barbara, CA, USA) following appropriate dilution with ultrapure water. The morphology of nanoparticles was observed by transmission electron microscopy (TEM) (JEM-1400plus; JEOL, Tokyo, Japan). Nanoparticles were diluted with ultrapure water and a drop was deposited on a copper grid followed by staining with $2 \%(\mathrm{w} / \mathrm{v})$ phosphotungstic acid. The samples were left to dry and examined.

\section{Intermolecular Interactions}

The spectrums of fourier transform infrared spectroscopy (FT-IR) (Thermo Scientific Nicolet iS20) were recorded and used to analyze molecular bonding formation between LCNs and baicalein. ${ }^{30}$ Differential scanning calorimetry (DSC) (TA Instruments Q200) and X-ray diffractometry (XRD) (Bruker D8 Advance) were employed to determine the crystalline state of free baicalein and baicalein encapsulated into LCNs.

\section{In vitro Stability}

The in vitro colloidal stability of nanoparticles at $32^{\circ} \mathrm{C}$ was qualitatively measured by Turbiscan Tower ${ }^{\circledR}$ (Formulaction, L'Union, France) using multiple light scattering. The baicalein solution and nanoparticles, including BA-LCNs and BPC-LCNs, were placed into a cylindrical glass cell and then scanned from bottom to top. The transmission intensity profiles as a function of position were acquired over a scan. The variation of average transmitted intensity $(\Delta \mathrm{T})$ and turbiscan stability index (TSI) calculated from the signal value of transmission light were employed as main parameters to evaluate the stability of the nanoparticle suspensions. ${ }^{14,31-33}$

The long-term storage stability of nanoparticles was monitored at $4{ }^{\circ} \mathrm{C}$ for 3 months. The appearance, particle 
size, zeta potentials, content, and entrapment efficiency were determined to evaluate the stability of nanoparticles.

\section{Entrapment Efficiency}

Entrapment efficiency of baicalein in LCNs was determined by a mini-column centrifugation method. The mini-column was prepared by filling Sephadex G50 into a $5 \mathrm{~mL}$ plastic syringe. Two hundred microlitres of nanoparticle suspensions were added onto the mini-column and centrifuged at $500 \mathrm{rpm}$ for 1 min. Subsequently, the mini-column was eluted three times with $0.5 \mathrm{~mL}$ of deionized water by centrifugation (1000 rpm for $1 \mathrm{~min}$ ). The eluent was collected and disrupted with ethanol. Finally, the encapsulated baicalein concentration in the collected eluent was determined using high-performance liquid chromatography (HPLC) (Agilent 1200; Agilent Technologies, Santa Clara, CA, USA). Entrapment efficiency $(\mathrm{EE} \%)$ was calculated using the following equations: $\mathrm{EE} \%=$ (Entrapped drug)/(Total drug) $\times 100 \%$.

\section{In vitro Release}

The in vitro release profile of baicalein from nanoparticles was assessed using dialysis membrane method. The release medium was $0.5 \%(\mathrm{w} / \mathrm{v})$ Tween-80 in phosphate buffer saline (PBS, $\mathrm{pH}$ 7.4). Baicalein-loaded nanoparticles $(1 \mathrm{~mL})$ were placed into a dialysis bag (MWCO cut-off 8-12 kDa) and immersed into $100 \mathrm{~mL}$ of release medium at $37^{\circ} \mathrm{C}$ on a shaker (100 rpm). One-milliliter aliquots of release medium were withdrawn and an equal volume of fresh release medium was added at predetermined time points. The accumulative released percentage of baicalein was determined by HPLC. Each sample analysis was performed in triplicate.

\section{Animals}

Male Sprague-Dawley rats weighing 190-210 g were supplied by Beijing Vital River Laboratory Animal Technology Co., Ltd. (Beijing, China). Male New Zealand rabbits were supplied by the Jinmuyang Laboratory Animal Breeding Co., LTD (Beijing, China). All animal experiments were approved by the Institutional Animal Care and Use Committee of Institute of Materia Medica, Chinese Academy of Medical Sciences \& Peking Union Medical College (No. 00005306). The care of laboratory animal and animal experimental operations were performed under the Beijing Administration Rule of Laboratory Animal.

\section{Ex vivo Permeation Study}

Male Sprague-Dawley rats were anesthetized using 2\% sodium pentobarbital $(60 \mathrm{mg} / \mathrm{kg})$ and their abdomen hair was carefully removed with an electric shaver. After being sacrificed by cervical dislocation, the abdomen skins were stripped from the hairless regions and the subcutaneous fat and connective tissue were scrapped off from the skins. Obtained skins were trimmed into appropriate size, washed with normal saline, blotted dry with filter paper, and then stored at $-20^{\circ} \mathrm{C}$ for future use. Integrity of the skins was checked to make sure that no skin damage occurred before the experiment.

Ex vivo permeation experiments were performed using horizontal Side-Bi-Side diffusion cells (PermeGear, Hellertown, PA, USA), in which the volume of supply pool, receiving pool, and the effective penetration area were $3.4 \mathrm{~mL}, 6 \mathrm{~mL}$, and $1.77 \mathrm{~cm}^{2}$, respectively (Figure $5 \mathrm{~A})$. Intact and thawed skin was embedded between the supply and receiving pool of the diffusion cell, with stratum corneum facing the supply pool. Baicaleinloaded nanoparticles were placed into the supply pool and the receiving pool was filled with $40 \%$ (w/v) PEG400 in normal saline (containing $1 \%$ vitamin C) which can meet a sink condition for baicalein. The receptor medium was maintained at a constant temperature of $32^{\circ} \mathrm{C}$ with no bubbles under constant stirring $(500 \mathrm{rpm})$ throughout the experiment. At predetermined time points $(2,4,6,8,10,20,22$, and $24 \mathrm{~h}), 1 \mathrm{~mL}$ of receptor medium was withdrawn and an equal volume of fresh receptor medium was added into the receiving pool. Further, the receptor medium was filtered through a $0.45 \mu \mathrm{m}$ membrane filter and analyzed for baicalein content using HPLC. At the end of experiments, the skins were taken out carefully, washed with fresh receptor medium, cut into pieces, and transferred into a centrifuge tube. Normal saline containing $5 \%$ vitamin $\mathrm{C}$ was added to the centrifuge tube and sonicated for $10 \mathrm{~min}$. Then, aliquots of homogenate were mixed well with methanol by vortexing and centrifuged at 14,000 rpm for $15 \mathrm{~min}$. The supernatant was collected and the content of baicalein was analyzed by HPLC.

\section{In vivo Permeation Study}

Male Sprague-Dawley rats were anesthetized using 2\% sodium pentobarbital $(60 \mathrm{mg} / \mathrm{kg})$ and their dorsal hair was carefully removed using an electric shaver and depilatory cream. The bare skins were washed with normal saline and the rats were allowed to recover for $24 \mathrm{~h}$. Integrity of the skins was checked to make sure that no skin damage occurred before the experiment. The self-made transdermal diffusion cell system was fixed on the dorsal bare skin with 
a neutral silicone sealant (Figure 6A), in which the effective penetration area was $2.8 \mathrm{~cm}^{2}$. Baicalein-loaded nanoparticles were placed into the diffusion pool which was sealed with a cover. Drug administration was sustained for $24 \mathrm{~h}$, and thereafter, the rats were sacrificed and the skin region that received transdermal treatment was harvested. Subsequent treatment of skin samples and extraction of baicalein from skin tissues were carried out according to the same methods described above. Baicalein content was analyzed by HPLC.

To investigate the mechanism of nanoparticles to enhance baicalein penetration through the skin barrier, the skin region that received transdermal treatment for 24 $\mathrm{h}$ was harvested, washed with normal saline, and blotted dry with filter paper. Skin tissues were fixed with $4 \%$ paraformaldehyde for $72 \mathrm{~h}$ and then embedded in paraffin for hematoxylin and eosin (HE) staining. Structure morphological changes of stratum corneum were observed under a microscope.

\section{Primary Skin Irritation Test}

To preliminarily evaluate the safety and biocompatibility of nanoparticles to skins, a skin irritation test was performed in rabbits as described before. ${ }^{34,35}$ The hair of a healthy male New Zealand rabbit was carefully removed using an electric shaver and depilatory cream. The bare skins were washed with normal saline and the rabbit was allowed to recover for $24 \mathrm{~h}$. Integrity of the skins was checked to make sure that no skin damage occurred before the experiment. Nanoparticle suspensions $(0.5 \mathrm{~mL})$ were spread evenly onto the test skin area and successively covered with gauze and sealing film to prevent their loss. The skin area only treated with normal saline was used as control. Further, it was wrapped with nonirritating tape. Drug administration was sustained for $4 \mathrm{~h}$, and thereafter, the skin region that received transdermal treatment was washed with normal saline. The dermal symptoms of erythema and edema at the application sites were carefully examined and scored based on the evaluation criteria at each observation period. Score ranges of $<0.5,0.5-2.0$, $2.0-6.0$, and $\geq 6.0$ were defined as no irritation, slight irritation, middle irritation, and strong irritation, respectively.

\section{Statistical Analysis}

All data subjected to statistical analyses were obtained from at least three parallel experiments. The statistical analyses were performed using unpaired two-tailed Student's $t$-test for two groups, and one-way ANOVA for multiple groups with GraphPad Prism version 7.00 for
Windows (GraphPad Software, La Jolla, CA, USA). A P value of $\leq 0.05$ was considered statistically significant.

\section{Results and Discussion Properties and Characteristics of Nanoparticles}

Transdermal delivery of therapeutic agents via LCNs holds great potential owing to the ability to encapsulate drugs and sustain their release, prolong the residence of the payloads within the skin, and effectively penetrate the skin barriers. Baicalein is a promising candidate for transdermal therapy of dermatosis due to its anti-inflammatory and anti-bacterial activities. However, poor hydrophilicity and lipophilicity of baicalein hinder its permeation into skin and decrease its entrapment efficiency in nanocarriers. Phospholipids have a long fatty acid chain (lipophilic part); therefore, the lipophilicity of drugs can be improved by the addition of phospholipids to form phospholipid complexes. In the present study, baicalein-phospholipid complex (BPC) was prepared for use as an intermediate of LCNs with enhanced lipophilicity. LCNs loaded with BPC were successfully developed and evaluated for transdermal penetration properties in vitro and in vivo.

In the present study, BPC-LCNs were successfully prepared by injection of ethanolic lecithin/BPC solution into an aqueous chitosan solution. Negatively charged lecithin interacted with the positively charged chitosan through self-assembly (Figure 1A). BPC-LCNs obtained were transparent and yellow. They were characterized based on mean particle size, size distribution (PdI), and zeta potential, which were $147.6 \mathrm{~nm}, 0.353$, and $+33.0 \mathrm{mV}$, respectively (Figure $1 \mathrm{~B}$ and $\mathrm{C}$ ). The high positive zeta potential is attributed to the cover of positively charged chitosan on the surface of the nanoparticles and contributes to the favorable stability of BPC-LCNs. The morphology of nanoparticles analyzed using TEM indicated that they were roughly spherical and subspherical characterized by a compact core surrounded by a transparent corona (Figure 1D). The hydrophilic outer shell was slightly corrugated, likely due to the dehydration of samples before the TEM examination.

High entrapment efficiency (EE\%) is beneficial to the stability of LCNs and is an essential prerequisite for effective delivery of drugs at the target site. The $\mathrm{EE} \%$ and content of baicalein in LCNs were $84.5 \pm 1.0 \%$ and 0.88 $\pm 0.04 \mathrm{mg} / \mathrm{mL}$, respectively. BPC significantly increased the lipophilicity of baicalein over 13-fold. The high 
A

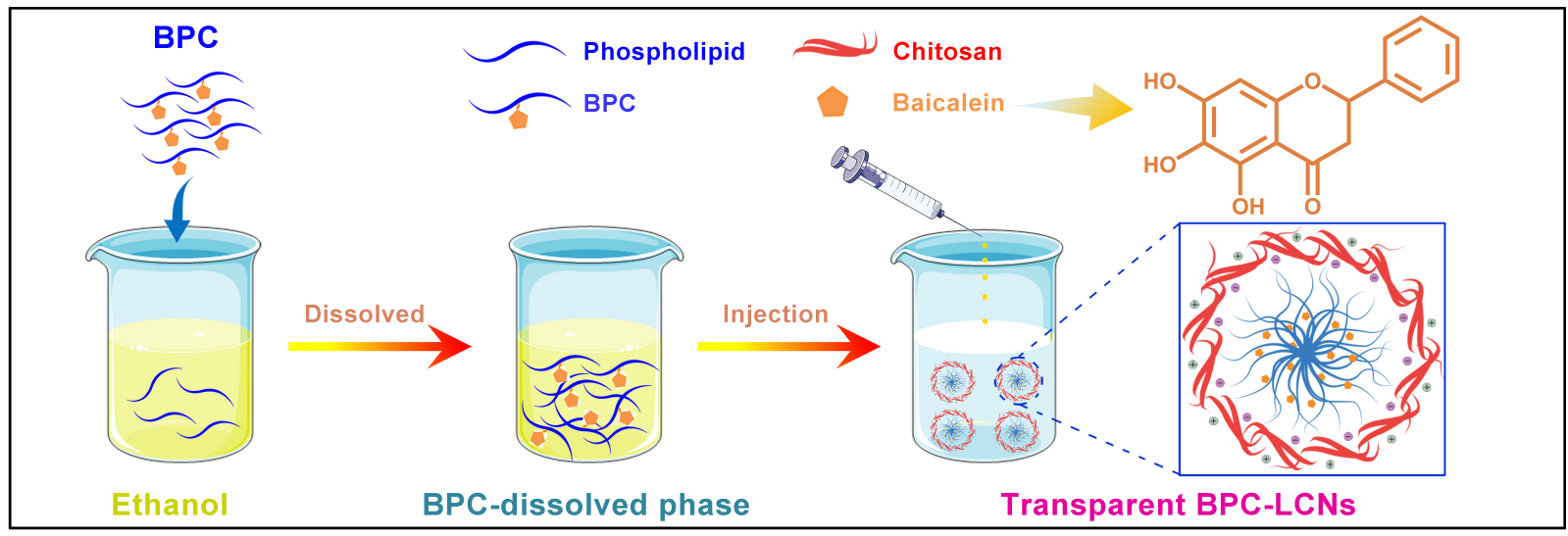

B

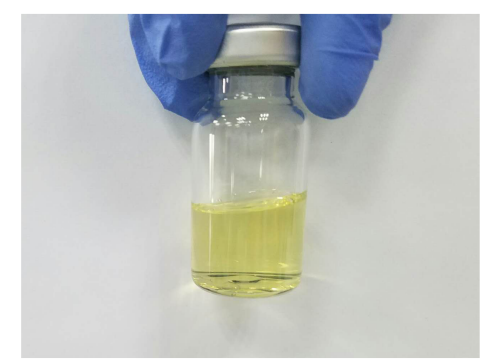

C

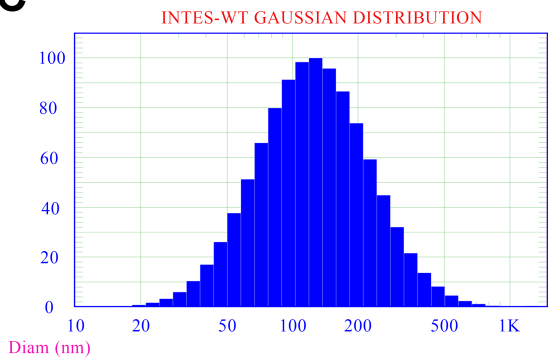

D

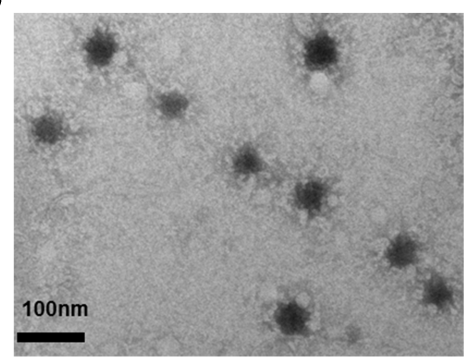

Figure I The preparation process and in vitro characterization of BPC-LCNs. (A) Schematic illustration of the preparation of BPC-LCNs. (B) The appearance, (C) particle size distribution, and (D) the morphology of BPC-LCNs.

Abbreviations: BPC, baicalein-phospholipid complex; BPC-LCNs, lecithin/chitosan nanoparticles loaded with baicalein-phospholipid complex.

entrapment efficiency may be attributed to the enhanced lipophilicity of baicalein by the preparation of BPC, which would be distributed evenly in the lipid core of LCNs.

\section{Intermolecular Interactions}

The possible intermolecular interactions between baicalein and phospholipids in the baicalein-phospholipid complex (BPC) were investigated by FT-IR. The FT-IR spectrums of pure baicalein, phospholipids, a physical mixture of baicalein and phospholipids, and BPC are shown in Figure 2A. The characteristic peaks of pure baicalein were $3411.6 \mathrm{~cm}^{-1}(\mathrm{O}-\mathrm{H}$ stretching) and $1657.4 \mathrm{~cm}^{-1}(\mathrm{C}=\mathrm{O}$ stretching). Phospholipid characteristic peaks included: $2922.6 \mathrm{~cm}^{-1}$ and $2852.9 \mathrm{~cm}^{-1}$ (C-H stretching band of long fatty acid chain), $1735.6 \mathrm{~cm}^{-1}$ ( $\mathrm{C}=\mathrm{O}$ stretching), $1241.8 \mathrm{~cm}^{-1}$ ( $\mathrm{P}=\mathrm{O}$ stretching), 1089.0 $\mathrm{cm}^{-1}$ (P-O-C stretching), and $968.0 \mathrm{~cm}^{-1}\left(\mathrm{~N}^{+}\left(\mathrm{CH}_{3}\right)_{3}\right.$ stretching). As expected, the physical mixture of baicalein and phospholipids exhibited the same vibrational frequencies of the individual components. Different from the physical mixture, the characteristic peak $(\mathrm{C}=\mathrm{O})$ of baicalein in $\mathrm{BPC}$ was red-shifted and the peak ( $\mathrm{O}-\mathrm{H}$ stretching) broadened. Additionally, the characteristic peak of the polar head of phospholipids was blue-shifted (from $1241.8 \mathrm{~cm}^{-1}$ to 1227.4 $\mathrm{cm}^{-1}$ ), whereas the characteristic peaks of $\mathrm{C}-\mathrm{H}$ stretching band of long fatty acid chain at $2922.7 \mathrm{~cm}^{-1}$ and 2852.7 $\mathrm{cm}^{-1}$ were also observed in BPC. These results imply that BPC formation did not produce new chemical bonds but weak physical interactions between baicalein and phospholipids, such as hydrogen bond or van der Waals forces. ${ }^{28}$

DSC and XRD analyses were performed to identify the crystalline state of pure baicalein, BPC, and BPC encapsulated into LCNs. ${ }^{36}$ The DSC thermogram of pure baicalein exhibits a sharp endothermic peak at $267.5^{\circ} \mathrm{C}$ (Figure 2B), corresponding to the melting point of the crystalline baicalein. On the contrary, the melting peak of the crystalline baicalein was not visible in the BPC curve, indicating that the crystalline state of pure baicalein transformed into amorphous form after interacting with phospholipid. Likewise, the DSC curve of BPCLCNs did not show the melting peak of baicalein, suggesting that the amorphous state of baicalein in BPC remained unchanged in LCNs. In addition, the characteristic absorption peak of pure baicalein was absent in the XRD curve of BPC and BPC-LCNs (Figure 2C), further demonstrating a disordered crystalline state of baicalein in BPC and LCNs. 

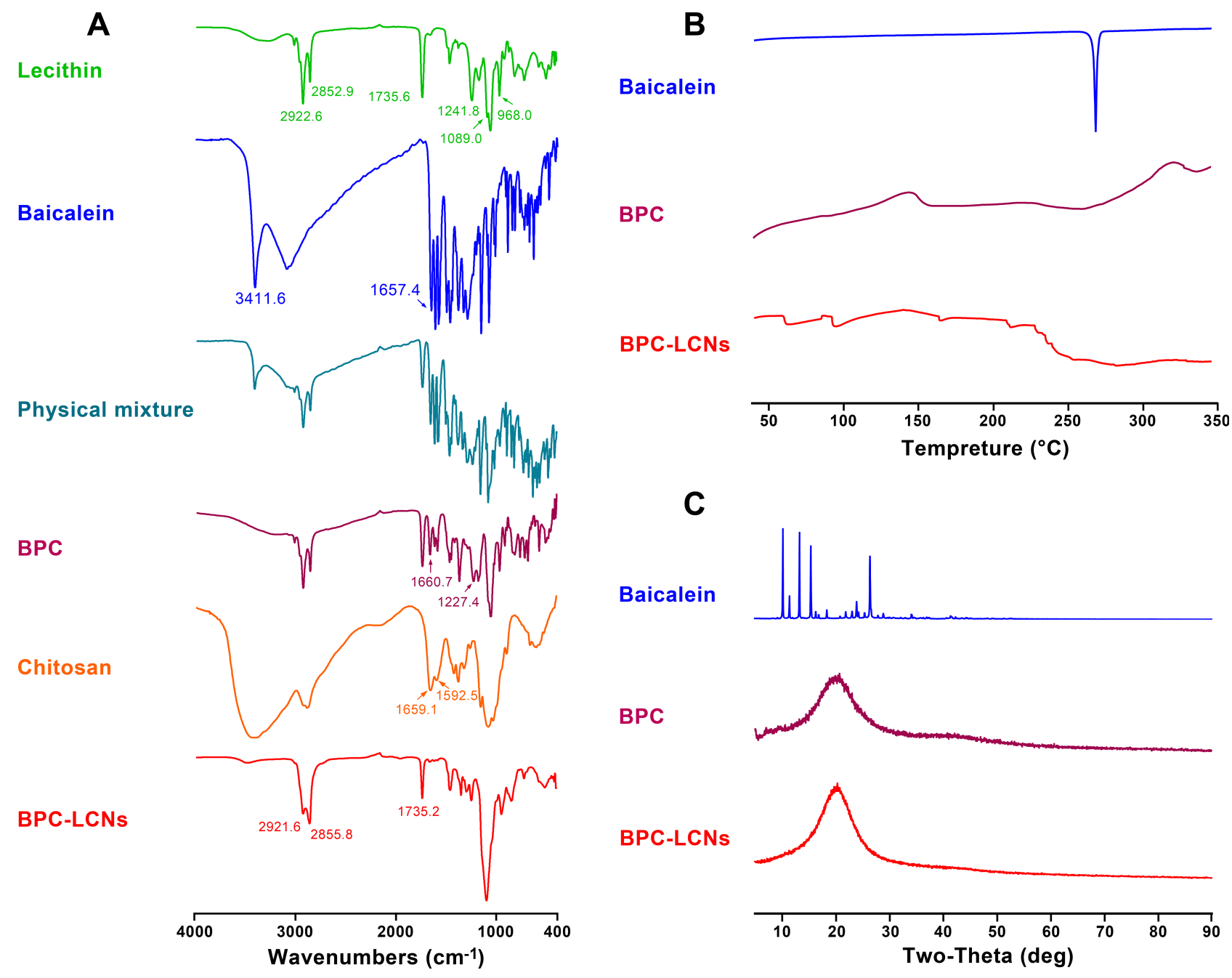

Figure 2 Intermolecular interactions in BPC-LCNs. (A) FT-IR spectrums of lecithin, baicalein, physical mixture of lecithin and baicalein, BPC, chitosan, and BPC-LCNs. (B) DSC thermograms and (C) XRD curves of baicalein, BPC, and BPC-LCNs.

Abbreviations: BPC-LCNs, lecithin/chitosan nanoparticles loaded with baicalein-phospholipid complex; FT-IR, fourier transform infrared spectroscopy; BPC, baicaleinphospholipid complex; DSC, differential scanning calorimetry; XRD, X-ray diffractometry.

These results confirmed that BPC had been successfully prepared and encapsulated into LCNs in amorphous form.

In order to explore the structure of LCNs, the interaction between the chitosan and the lecithin was investigated by FTIR. ${ }^{37}$ As shown in Figure 2A, two characteristic peaks of chitosan at $1659.1 \mathrm{~cm}^{-1}$ and $1592.5 \mathrm{~cm}^{-1}$ were assigned to the carbonyl $(\mathrm{C}=\mathrm{O})$ stretching (amide $\mathrm{I})$ and $\mathrm{N}-\mathrm{H}$ bending vibration (amide II), respectively. The spectrum of lyophilized LCNs shows the typical absorption bands of lecithin, the main component of the nanoparticles. In the BPC-LCNs spectrum, the intensity of the absorption band at $1735.6 \mathrm{~cm}^{-1}$ decreased with respect to lecithin due to the stretching of the carbonyl groups of the fatty acids. In addition, the characteristic peak of $\mathrm{N}-\mathrm{H}$ bending vibration of chitosan at $1592.5 \mathrm{~cm}^{-1}$ disappeared in the BPC-LCNs spectrum. These results indicate that ionic interactions may form between phosphate groups of lecithin and amino groups of chitosan. $^{17}$

\section{In vitro Stability of Nanoparticles}

The colloidal stability and the long-term storage stability have been recognized as two significant factors to be taken into account to propose nanoparticles as promising carriers for the efficient delivery of therapeutic agents. ${ }^{38,39}$

The colloidal stability of nanoparticles, including BALCNs and BPC-LCNs, were assessed using the Turbiscan $\mathrm{Lab}^{\circledR}$ Expert, an advanced analytical instrument that can identify early imperceptible changes before the appearance of macroscopic physical modifications to nanoparticle suspensions. ${ }^{40}$ As baicalein was poorly soluble in water, the baicalein solution was chosen to be a control of unstable systems (Figure 3A and D). The variation of the droplet 

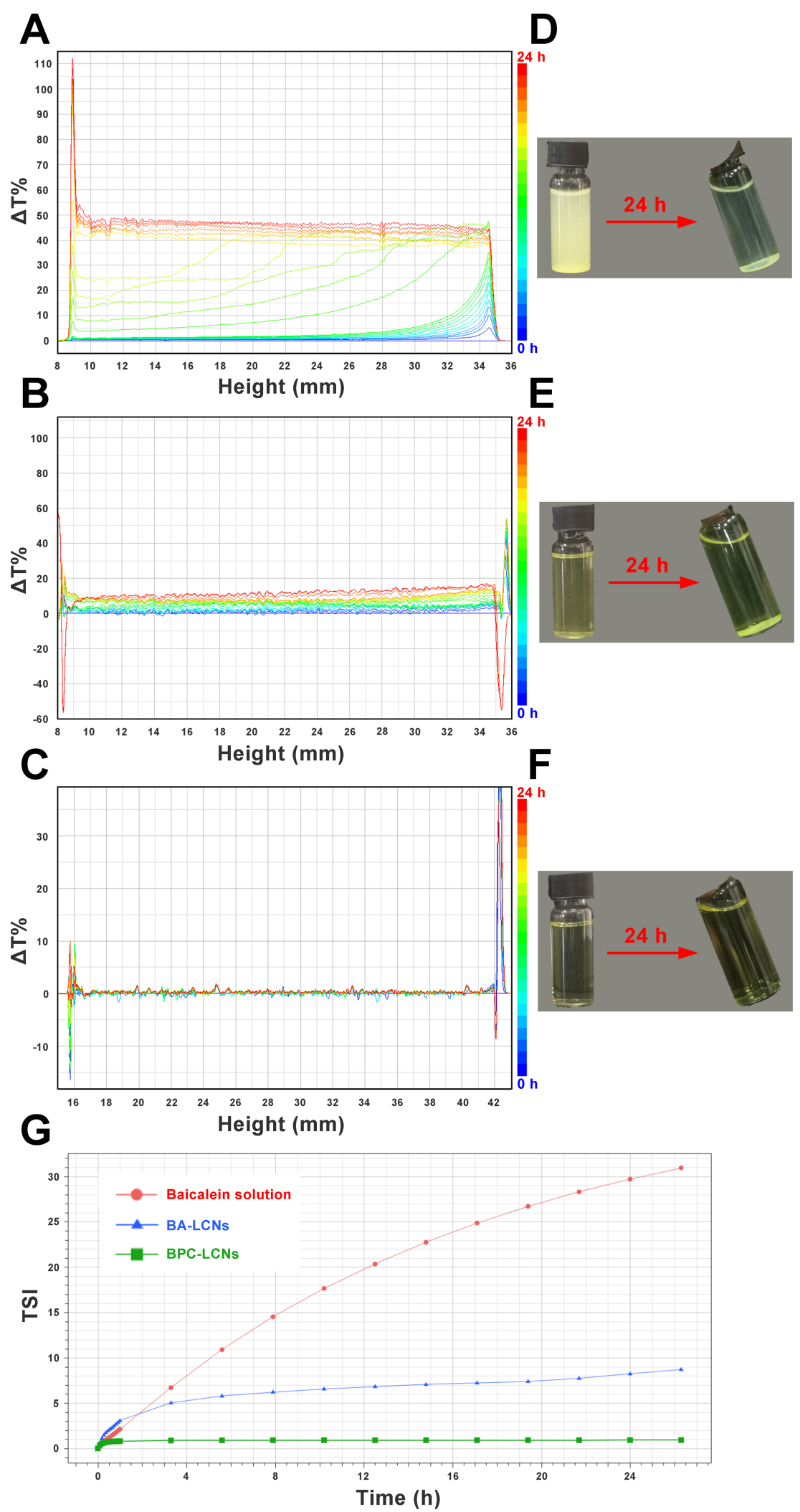

Figure 3 In vitro colloidal stability of BPC-LCNs. Variations of transmission profiles $(\triangle T)$ of $($ A) baicalein solution, (B) BA-LCNs, and (C) BPC-LCNs dispersed in distilled water at $32^{\circ} \mathrm{C}$ for $24 \mathrm{~h}$. The appearance of (D) baicalein solution, (E) BA-LCNs, and (F) BPC-LCNs dispersed in distilled water at $32^{\circ} \mathrm{C}$ for $24 \mathrm{~h}$. (G) TSI of baicalein solution, BA-LCNs, and BPC-LCNs dispersed in distilled water at $32^{\circ} \mathrm{C}$ for $24 \mathrm{~h}$.

Abbreviations: BPC-LCNs, lecithin/chitosan nanoparticles loaded with baicalein-phospholipid complex; BA-LCNs, lecithin/chitosan nanoparticles loaded with baicalein; TSI, turbiscan stability index. 
Table I The Long-Term Storage Stability of BPC-LCNs at $4^{\circ} \mathrm{C}$

\begin{tabular}{|l|l|l|l|l|l|l|}
\hline $\begin{array}{l}\text { Temperature } \\
\left({ }^{\circ} \mathbf{C}\right)\end{array}$ & $\begin{array}{l}\text { Time } \\
(\text { Month) }\end{array}$ & $\begin{array}{l}\text { Diameter } \\
(\mathbf{n m})\end{array}$ & $\begin{array}{l}\text { Zeta Potential } \\
(\mathbf{m V})\end{array}$ & Content $(\mathbf{m g} / \mathbf{m L})$ & EE (\%) & Appearance \\
\hline 4 & 0 & 186.6 & +33.08 & 0.91 & 83.4 & Yellowish transparent \\
& 1 & 183.8 & +33.15 & 0.92 & 83.7 & Yellowish transparent \\
& 3 & 186.0 & +32.89 & 0.91 & 82.6 & Yellowish transparent \\
\hline
\end{tabular}

volume fraction (migration) or size (coalescence) was detected as a variation of light transmission $(\Delta \mathrm{T})$ profiles. It is generally considered that variations higher than $10 \%$ are representative of an unstable formulation. ${ }^{33,41}$ As shown in Figure $3 \mathrm{C}$, the $\triangle \mathrm{T}$ of BPC-LCNs was extremely low and less than $10 \%$, suggesting that there was no aggregation or sedimentation when BPC-LCNs were left for $24 \mathrm{~h}$ (Figure 3F). On the contrary, sedimentation occurred for BA-LCNs, as evidenced by the higher $\Delta \mathrm{T}$ (above $10 \%$ ) value and by their appearance with obvious precipitation (Figure $3 \mathrm{~B}$ and $\mathrm{E}$ ). Apart from $\Delta \mathrm{T}$, the TSI values were calculated from the changes in transmitted light to compare the stability more intuitively. Higher TSI values represent less stability. ${ }^{42}$ Consistent with the results of $\Delta \mathrm{T}$, the TSI of BA-LCNs increased with time, while BPC-LCNs maintained relatively low TSI values (Figure 3G). This superior colloidal stability could be in favor of BPC-LCNs to improve the transdermal retention and permeability of baicalein.

The long-term storage stability of BPC-LCNs was evaluated by monitoring the changes in appearance, particle size, zeta potential, content, and entrapment efficiency over three months at $4^{\circ} \mathrm{C}$. The result showed that all the monitoring indexes were hardly changed within three months, indicating that BPC-LCNs exhibited good stability (Table 1). These results indicated that BPC-LCNs exhibited favorable colloidal and long-term storage stability, which may be ascribed to the integrative effect of high positive zeta potential and high entrapment efficiency.

\section{In vitro Sustained-Release Profile of Nanoparticles}

The in vitro release profiles of baicalein and BPC-LCNs are shown in Figure 4. Compared with the rapid release feature of free baicalein (BA) (about 80\% within 4 h), BPC-LCNs exhibited a sustained-release profile with the cumulative release of only $28 \%$ within $4 \mathrm{~h}$. The results suggest that LCNs effectively encapsulate the lipophilic intermediate BPC, and allows sustained-release of baicalein. Notably, no initial burst release was observed over a $24 \mathrm{~h}$ period, which may be attributed to the presence of amorphous BPC at the lipid core and its absence on the surface. ${ }^{18,43}$ The sustainedrelease characteristic of BPC-LCNs, which may be associated with the slower diffusion of baicalein from LCNs, could prevent the spreading of baicalein over the stratum corneum, thus ensuring to effective delivery.

\section{Enhanced Penetration of Nanoparticles ex vivo}

The poor hydrophilicity and lipophilicity of baicalein prevent it from being topically applied using conventional formulations. Nanoparticles prepared with biocompatible and bioadhesive materials are desirable for topical and transdermal drug delivery. Chitosan has been reported to possess bioadhesive and penetration enhancing properties which enable it to be frequently used in transdermal and transmucosal delivery. Positively charged BPC-LCNs obtained via the selfassembled interaction of negatively charged lecithin and positively charged chitosan can interact effectively with the negatively charged skin. These interactions prolong the retention of BPC-LCNs on the skin and then facilitate the penetration of nanoparticles across the skin barrier.

To evaluate the potential of LCNs in delivering baicalein across the skin, the ex vivo skin permeation study on the excised rat skin was carried out using horizontal SideBi-Side cells (Figure 5A). In this experiment, the

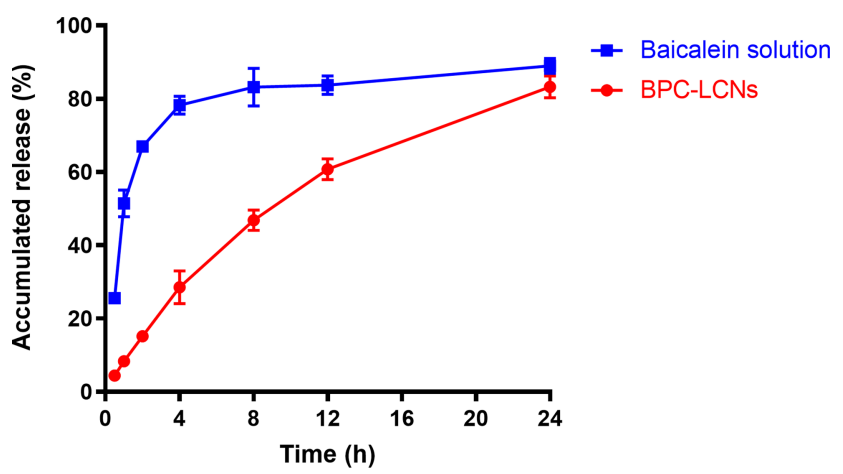

Figure 4 In vitro release profile of BPC-LCNs in PBS ( $\mathrm{pH} 7.4)$ containing $0.5 \%(\mathrm{w} / \mathrm{v})$ Tween 80. Each value represents the mean \pm SD $(n=3)$.

Abbreviations: BPC-LCNs, lecithin/chitosan nanoparticles loaded with baicaleinphospholipid complex; PBS, phosphate buffer saline. 
A

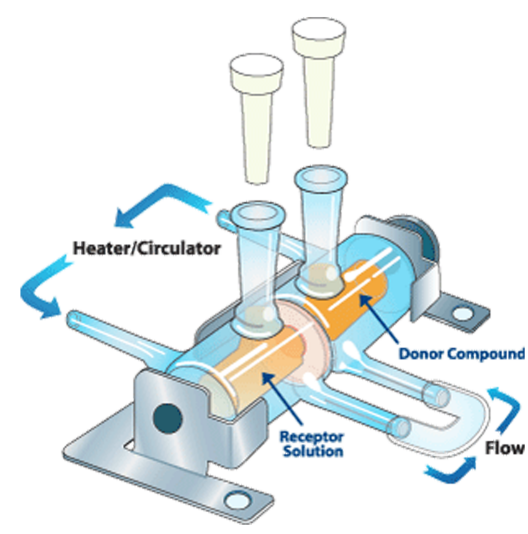

B

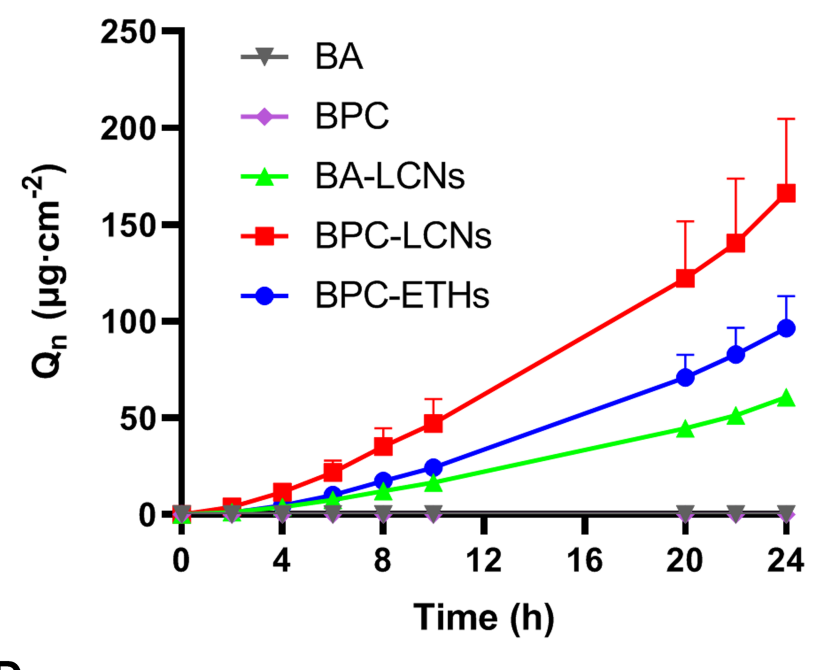

C

D

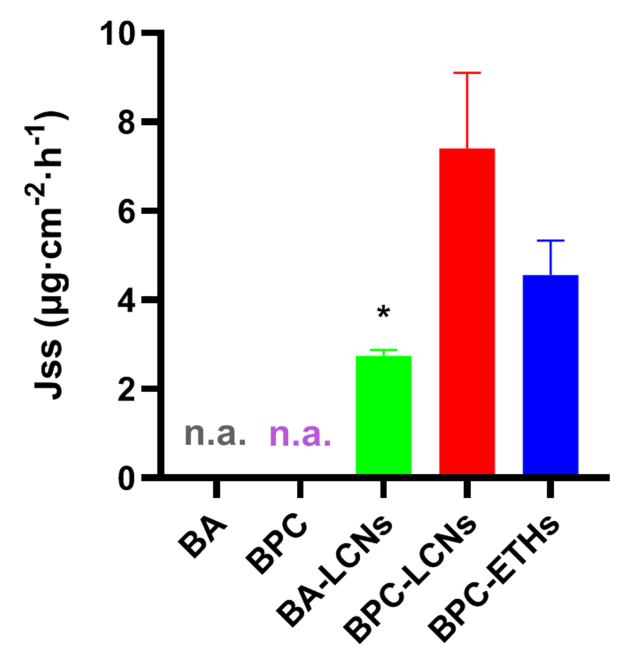

E
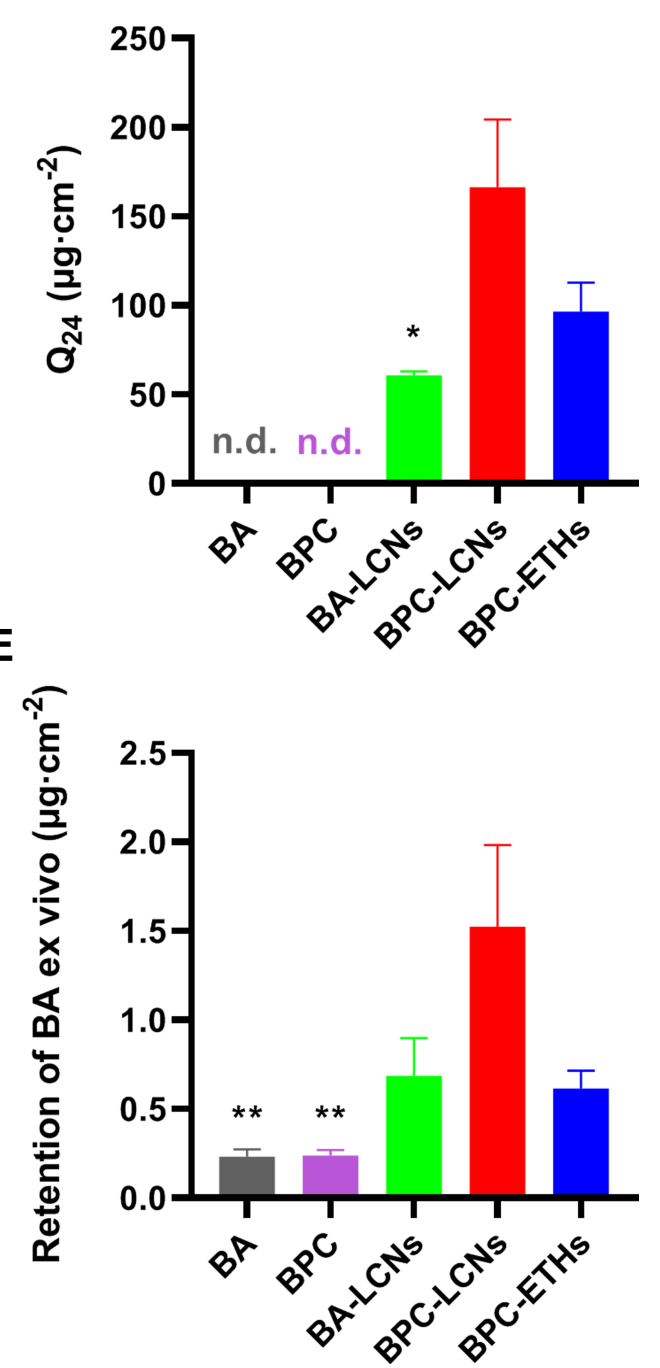

Figure 5 Ex vivo permeation profiles of nanoparticles. (A) The schematic diagram of a Side-bi-Side diffusion cell, (B) the accumulative permeation amount $\left(\mathrm{Q}_{\mathrm{n}}\right)$ of baicalein at the predetermined time points, (C) the accumulative permeation amount $\left(Q_{24}\right)$ of baicalein at 24 h, (D) the permeation rate (Jss) of baicalein, (E) the accumulative retention amounts of baicalein in the skins. Each value is represented as the mean \pm SEM $(n=4-5)$. *p $<0.05$ vs BPC-LCNs, **p $<0.01$ vs BPC-LCNs.

Abbreviations: BA, baicalein; BPC, baicalein-phospholipid complex; BA-LCNs, lecithin/chitosan nanoparticles loaded with baicalein; BPC-LCNs, lecithin/chitosan nanoparticles loaded with baicalein-phospholipid complex; BPC-ETHs, ethosomes loaded with baicalein-phospholipid complex; n.d., not detected; n.a., not applicable. 
permeation study of free baicalein (BA), baicaleinphospholipid complex (BPC), baicalein-loaded LCNs (BA-LCNs), and BPC-loaded ethosomes (BPC-ETHs) was performed and compared with that of baicaleinphospholipid complex-loaded LCNs (BPC-LCNs). Permeability of BPC-LCNs was assessed by calculating the accumulative permeation amount $\left(Q_{n}\right)$ and the permeation rate (Jss) of baicalein through the skin into the receiving pool. The $\mathrm{Q}_{\mathrm{n}}$ of baicalein across the excised rat skin via various formulations was plotted as a function of time (Figure 5B). As shown in Figure 5B, baicalein was not detected in the receiving pool for $24 \mathrm{~h}$ after administration of free baicalein, indicating that free baicalein did not penetrate through the rat skin, which may be ascribed to poor hydrophilicity and lipophilicity reported previously. After encapsulation in nanocarriers, baicalein could penetrate through the rat skin into the receiving pool at just $2 \mathrm{~h}$, demonstrating that nanocarriers can significantly facilitate the penetration of baicalein across the skin barrier (Figure 5B). Notably, the $\mathrm{Q}_{\mathrm{n}}$ and Jss of baicalein from BPC-LNCs were both significantly higher in comparison with that from BA-LCNs (Figure 5B and D). The $\mathrm{Q}_{\mathrm{n}}$ of baicalein from BPC-LNCs at $24 \mathrm{~h}$ was $166.3 \pm 76.6 \mu \mathrm{g} \cdot \mathrm{cm}^{-2}$, almost 2.7 times higher than that from BA-LCNs with a value of $60.7 \pm 4.8 \mu \mathrm{g} \cdot \mathrm{cm}^{-2}$ (Figure 5C). In addition, the Jss of baicalein from BPCLNCs $\left(7.4 \pm 3.4 \mu \mathrm{g} \bullet \mathrm{cm}^{-2} \cdot \mathrm{h}^{-1}\right)$ was approximately 2.7 -fold higher than that from BA-LCNs $\left(2.7 \pm 0.3 \mu \mathrm{g} \cdot \mathrm{cm}^{-2} \cdot \mathrm{h}^{-1}\right)$ (Figure 5D). Compared to BA-LCNs, the enhanced permeability of BPC-LCNs could be attributed to the different lipophilicity of encapsulated agents, free baicalein and BPC. As mentioned above, BPC could significantly enhance the lipophilicity of baicalein (almost 13.4-fold), which enabled it to distribute easily and evenly in the lipid core of LCNs, thereby improving the stability and permeability of LCNs through the skin barrier. Therefore, the superior permeability of BPC-LCNs in the skin will be beneficial in enhancing the lipophilicity of encapsulated agents, leading to improved therapeutic efficacy.

Ethosomes and transfersomes are specially tailored lipid vesicles and generally recognized as promising nanocarriers in transdermal delivery of encapsulated agents. These two vesicular colloidal carriers improve skin permeability significantly compared with other lipid nanocarriers due to the favorable flexibility and deformability conferred by the presence of edge-activator agents (ie, ethanol and sodium cholate), which is different from the mechanism underlying LCNs' enhanced penetration effect. ${ }^{44-48}$ As expected, BPC-loaded ethosomes (BPC-ETHs) significantly improved the transdermal permeability of baicalein compared with free baicalein and BPC, as shown by the high values of $Q_{n}$ and Jss. Even more noteworthy is that BPC-LCNs exhibited a trend of enhanced permeability as compared to BPC-ETHs, which may be attributed to the enhanced penetration effect of chitosan which changed the morphology of the stratum corneum and opened the tight conjugation of the corneocyte layers. ${ }^{49,50}$ The results

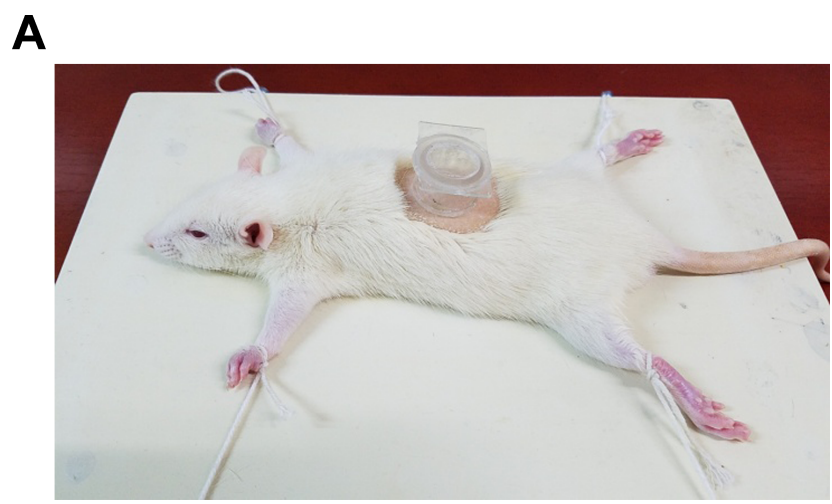

B

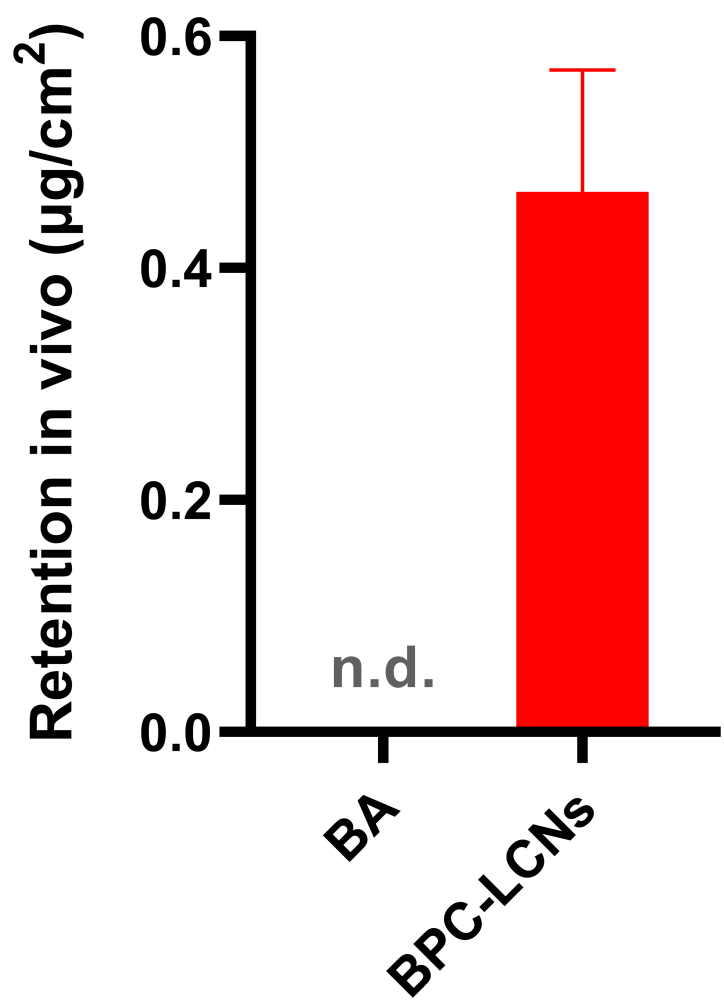

Figure 6 In vivo permeation profiles of nanoparticles. (A) In vivo topical administration device, (B) the accumulative retention amounts of baicalein in the skins after $24 \mathrm{~h}$. Each value is represented as the mean $\pm \operatorname{SEM}(n=5)$.

Abbreviations: BA, baicalein; BPC-LCNs, lecithin/chitosan nanoparticles loaded with baicalein-phospholipid complex; n.d., not detected. 
further highlight the potential of BPC-LCNs in the effective delivery of baicalein across skin.

In addition to the accumulative permeation amount $\left(\mathrm{Q}_{\mathrm{n}}\right)$ and the permeation rate (Jss), the drug accumulated in the skin ex vivo was measured at the end of $24 \mathrm{~h}$. The amounts of baicalein accumulating in the skin were normalized by the area of skin and expressed as $\mu \mathrm{g}$ baicalein per $\mathrm{cm}^{2}$ of skin. As shown in Figure 5E, BPC-LCNs produced the highest accumulative amounts of baicalein in the skin within $24 \mathrm{~h}$ of administration in comparison with the other formulations, which may be attributed to the synergistic effect of the bioadhesive of chitosan and the lipophilicity of BPC.

\section{Improved Retention of Nanoparticles in vivo}

Accumulated amounts of baicalein in the skin $24 \mathrm{~h}$ after administration of BPC-LCNs were further investigated in vivo (Figure 6A). Consistent with the results of ex vivo permeation study, baicalein was not detected in the skin tissue for $24 \mathrm{~h}$ after topical application of free baicalein. Interestingly, BPC-LCNs significantly improved the retention of baicalein in the skin tissue at the same administration time with a value of $0.47 \pm 0.24 \mu \mathrm{g} \cdot \mathrm{cm}^{-2}$ (Figure 6B). This implies that LCNs based on phospholipid complex exhibit great application potential for the transdermal delivery of baicalein. Based on the ex vivo and in vivo results, improved retention of baicalein in the skin could be attributed to the enhanced lipophilicity of BPC causing LCNs to encapsulate baicalein effectively and sustain its release, and the compact cover of positively charged chitosan on LCNs strengthening the electrostatic interaction with the negatively charged skin, thereby extending the retention of baicalein in the skin. Other properties of LCNs, such as the nano-size and the large surface area, provide an excellent contact surface between the nanoparticles and skin and promote permeation and accumulation of baicalein in the skin. ${ }^{49}$

\section{The Mechanism to Promote Penetration}

Positively charged chitosan interacts with the negatively charged stratum corneum, thereby changing the morphology of the stratum corneum and loosening the close conjugation of corneocyte layers. ${ }^{50}$ To explore the topical effect of BPC-LCNs on the skin, the histopathology examination of untreated skin, skin treated with baicalein PEG/ ethanol solution or BPC-LCNs was performed using HE staining. Visually analysis showed that the untreated skin was characterized with compact stratum corneum with tight junctions, while the skin treated with baicalein
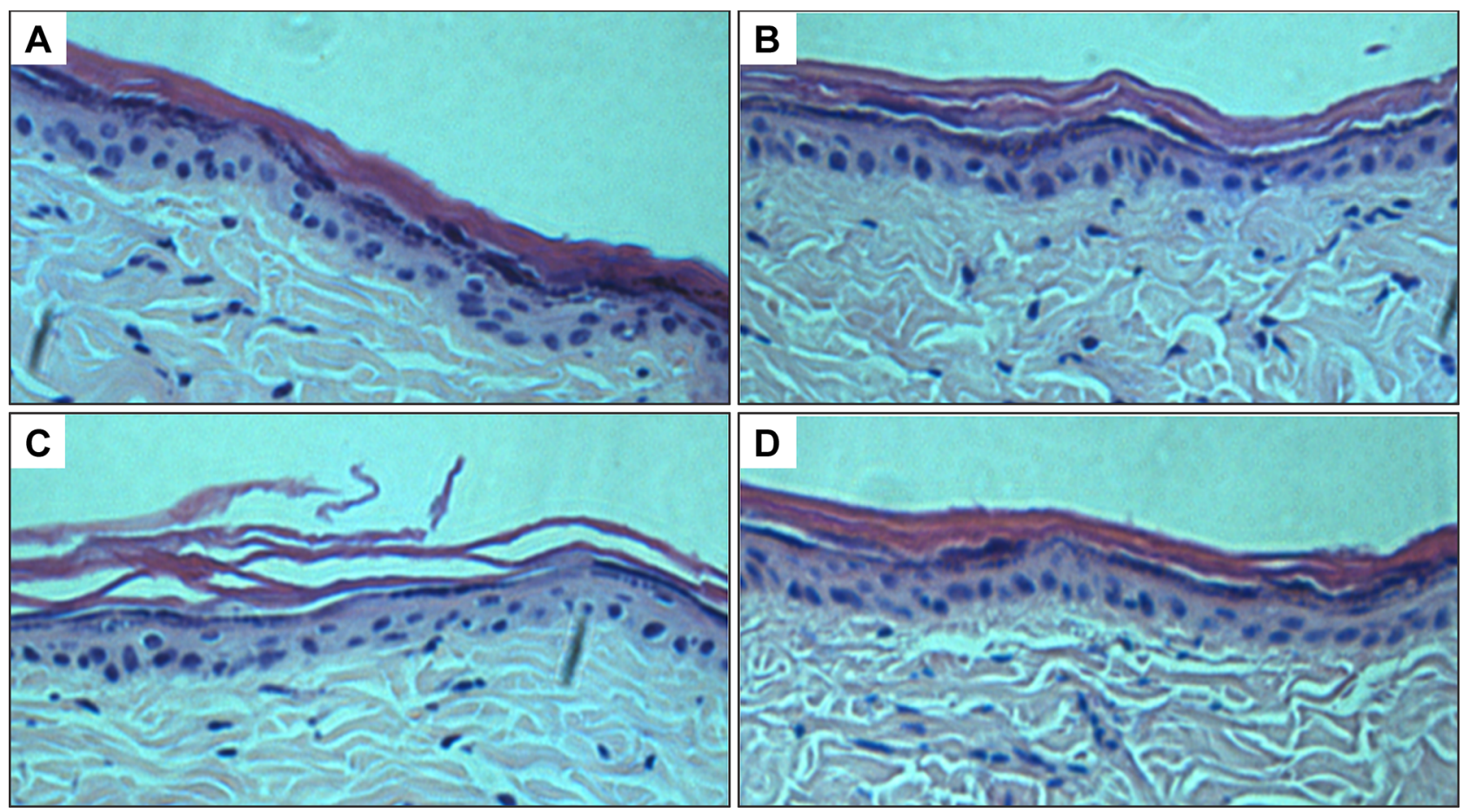

Figure 7 Histopathological photomicrographs $(\times 200)$ of the vertical section of rat skin after HE staining: $($ A) untreated skin, $($ B $)$ skin treated with baicalein PEG/ethanol solution, (C) skin treated with BPC-LCNs, and (D) recuperative skin after the removal of BPC-LCNs.

Abbreviations: HE, hematoxylin and eosin; PEG, polyethylene glycol; BPC-LCNs, lecithin/chitosan nanoparticles loaded with baicalein-phospholipid complex. 
PEG/ethanol solution exhibited a slight loose structure within the stratum corneum (Figure 7A and B). Ethanol can extract some of the lipid fraction from the stratum corneum and disrupt the organization of the lipid bilayer of stratum corneum. ${ }^{51}$ Specifically, skin treated with BPCLCNs for $24 \mathrm{~h}$ was characterized by a swollen, scattered, and loose stratum corneum which was thickened (Figure 7C). Of note, the compact stratum corneum with tight junctions recovered well after the removal of BPC-LCNs from the skin (Figure 7D), indicating that the effect of BPC-LCNs on the morphology of the stratum corneum was reversible. The reversibility of the stratum corneum ensures the safety of BPC-LCNs in clinical applications. These results proved that BPC-LCNs reversibly weakened the barrier function of the stratum corneum, thereby promoting drug accumulation and permeation.

\section{Primary Skin Irritation Test in vivo}

The effect of BPC-LCNs on the skin demonstrated that the barrier function of the stratum corneum was disturbed by topical application of BPC-LCNs. The barrier function of skin

A

\begin{tabular}{ccccccc}
\hline \multirow{2}{*}{ Group } & \multicolumn{5}{c}{ Time (h) } & \multirow{2}{*}{ Score } \\
\cline { 2 - 5 } & 0 & 1 & 2 & 3 & 4 & 0 \\
\hline Normal saline & 0 & 0 & 0 & 0 & 0 & 0 \\
BPC-LCNs & 0 & 0 & 0 & 0 & 0 & 0 \\
\hline
\end{tabular}

B

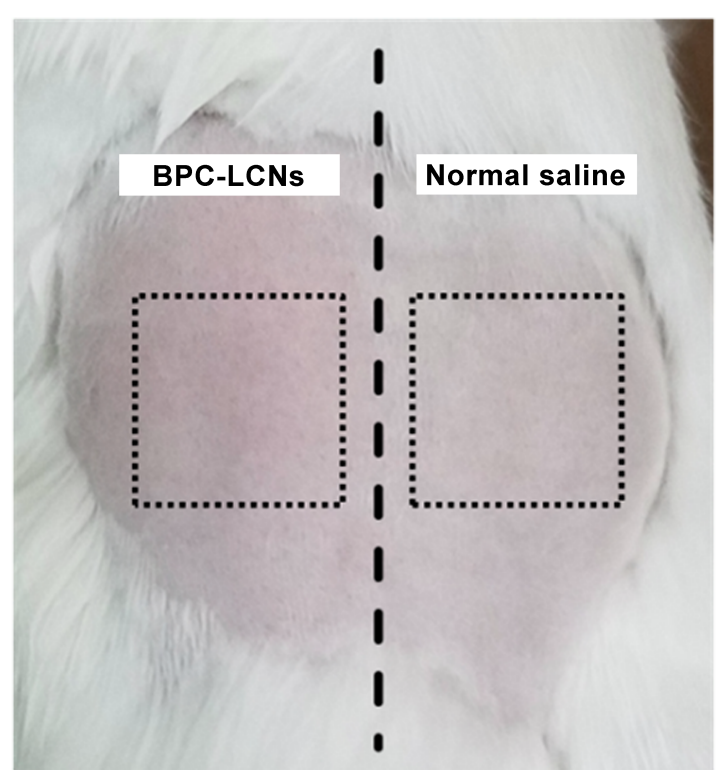

Figure 8 Rabbit skin irritation after $4 \mathrm{~h}$ of treatment with normal saline and BPCLCNs. (A) Scores of skin irritation test, (B) the photograph of the dorsal skin of rabbit exposed to normal saline and BPC-LCNs for $4 \mathrm{~h}$.

Abbreviation: BPC-LCNs, lecithin/chitosan nanoparticles loaded with baicaleinphospholipid complex. plays a pivotal role in preventing skin irritation. Therefore, the skin irritation test of BPC-LCNs was performed to preliminarily evaluate the safety of topical application of BPC-LCNs. As shown in Figure 8A, all scores of the erythema and edema were 0 at each observation period, demonstrating that no symptoms of erythema and edema occurred in BPC-LCNs-treated group throughout the experiment (Figure 8B). Based on the symptoms and calculated scores, the skin irritation intensity following BPC-LCNs treatment was considered as no irritation in skin, indicating that BPC-LCNs exhibited excellent biocompatibility. The results also indirectly proved the good biocompatibility of empty LCNs and BPC. The systematic comparison study, including normal saline, BPC, empty LCNs, and BPC-LCNs, will be further investigated in our future work.

\section{Conclusion}

In this study, LCNs based on phospholipid complex (BPCLCNs) were developed through a self-assembly interaction of positively charged chitosan and negatively charged lecithin. The BPC-LCNs efficiently encapsulated baicalein (EE, 84.5\%) and provided good transdermal retention and permeability of baicalein. They also conferred favorable stability, sustained drug release profile, prolonged the retention of baicalein at the skin, and efficient penetration through the barrier of stratum corneum without displaying skin irritation. Based on these findings, drugphospholipid complexes present a promising lipophilic intermediate to significantly enhance the entrapment efficiency of poorly lipophilic drugs in LCNs. More importantly, the application potential of LCNs as a superior transdermal delivery platform could be effectively expanded by combining the strategy of drug-phospholipid complex.

\section{Funding}

This work was financially supported by the National Science and Technology Major Project of China (Grant No. 2018ZX09721003 and 2018ZX09711001), the CAMS Innovation Fund for Medical Sciences (CIFMS) (201912M-1-005), and the Fundamental Research Funds for the Central Universities (Grant No. 3332019069).

\section{Disclosure}

The authors report no conflicts of interest for this work.

\section{References}

1. Ma Q, Gao Y, Sun W, et al. Self-Assembled chitosan/phospholipid nanoparticles: from fundamentals to preparation for advanced drug delivery. Drug Deliv. 2020;27(1):200-215. doi:10.1080/107175 44.2020.1716878 
2. Bugnicourt L, Ladavière C. A close collaboration of chitosan with lipid colloidal carriers for drug delivery applications. J Control Release. 2017;256:121-140. doi:10.1016/j.jconrel.2017.04.018

3. Alavi S, Haeri A, Dadashzadeh S. Utilization of chitosan-caged liposomes to push the boundaries of therapeutic delivery. Carbohydr Polym. 2017;157:991-1012. doi:10.1016/j.carbpol.2016.10.063

4. Mohammed MA, Syeda JTM, Wasan KM, Wasan EK. An overview of chitosan nanoparticles and its application in non-parenteral drug delivery. Pharmaceutics. 2017;9:4. doi:10.3390/pharmaceutics9040053

5. Liang J, Yan H, Puligundla P, Gao X, Zhou Y, Wan X. Applications of chitosan nanoparticles to enhance absorption and bioavailability of tea polyphenols: A review. Food Hydrocoll. 2017;69:286-292. doi:10.1016/j.foodhyd.2017.01.041

6. Lee EH, Lim SJ, Lee MK. Chitosan-coated liposomes to stabilize and enhance transdermal delivery of indocyanine green for photodynamic therapy of melanoma. Carbohydr Polym. 2019;224:115143. doi:10.1016/j.carbpol.2019.115143

7. Hadidi M, Pouramin S, Adinepour F, Haghani S, Jafari SM. Chitosan nanoparticles loaded with clove essential oil: characterization, antioxidant and antibacterial activities. Carbohydr Polym. 2020;236:116075. doi:10.1016/j.carbpol.2020.116075

8. Cosco D, Failla P, Costa N, et al. Rutin-loaded chitosan microspheres: characterization and evaluation of the anti-inflammatory activity. Carbohydr Polym. 2016;152:583-591. doi:10.1016/j.carbpol.2016.06.039

9. Iannone M, Mare R, Paolino D, et al. Characterization and in vitro anticancer properties of chitosan-microencapsulated flavan-3-ols-rich grape seed extracts. Int J Biol Macromol. 2017;104:1039-1045. doi:10.1016/j.ijbiomac.2017.07.022

10. Chaves LL, Silveri A, Vieira ACC, et al. pH-responsive chitosan based hydrogels affect the release of dapsone: design, set-up, and physicochemical characterization. Int $J$ Biol Macromol. 2019;133:1268-1279. doi:10.1016/j.ijbiomac.2019.04.178

11. Pawar KR, Babu RJ. Lipid materials for topical and transdermal delivery of nanoemulsions. Crit Rev Ther Drug Carrier Syst. 2014;31 (5):429-458. doi:10.1615/CritRevTherDrugCarrierSyst.2014010663

12. De Rose RF, Cristiano MC, Celano M, et al. PDE5 inhibitors-loaded nanovesicles: physico-chemical properties and in vitro antiproliferative activity. Nanomaterials. 2016;6(5):92. doi:10.3390/nano6050092

13. Cristiano MC, Cosco D, Celia C, et al. Anticancer activity of all-trans retinoic acid-loaded liposomes on human thyroid carcinoma cells. Colloid Surf B-Biointerfaces. 2017;150:408-416. doi:10.1016/j. colsurfb.2016.10.052

14. Di Francesco M, Primavera R, Fiorito S, et al. Acronychiabaueri analogue derivative-loaded ultradeformable vesicles: physicochemical characterization and potential applications. Planta Med. 2017;83(05):482-491. doi:10.1055/s-0042-112225

15. Senyigit T, Sonvico F, Rossi A, et al. In vivo assessment of clobetasol propionate-loaded lecithin-chitosan nanoparticles for skin delivery. Int J Mol Sci. 2016;18:1. doi:10.3390/ijms18010032

16. Senyigit T, Sonvico F, Barbieri S, Ozer O, Santi P, Colombo P. Lecithin/chitosan nanoparticles of clobetasol-17-propionate capable of accumulation in pig skin. $J$ Control Release. 2010;142 (3):368-373. doi:10.1016/j.jconrel.2009.11.013

17. Sonvico F, Cagnani A, Rossi A, et al. Formation of self-organized nanoparticles by lecithin/chitosan ionic interaction. Int $J$ Pharm. 2006;324(1):67-73. doi:10.1016/j.ijpharm.2006.06.036

18. Ozcan I, Azizoglu E, Senyigit T, Ozyazici M, Ozer O. Enhanced dermal delivery of diflucortolone valerate using lecithin/chitosan nanoparticles: in-vitro and in-vivo evaluations. Int $J$ Nanomed. 2013;8:461-475. doi:10.2147/IJN.S40519

19. Hafner A, Durrigl M, Pepic I, Filipovic-Grcic J. Short- and long-term stability of lyophilised melatonin-loaded lecithin/chitosan nanoparticles. Chem Pharm Bull. 2011;59(9):1117-1123. doi:10.1248/cpb.59.1117
20. Markovic M, Ben-Shabat S, Keinan S, Aponick A, Zimmermann EM, Dahan A. Prospects and challenges of phospholipid-based prodrugs. Pharmaceutics. 2018;10:4. doi:10.33 90/pharmaceutics 10040210

21. Lu M, Qiu Q, Luo X, et al. Phyto-phospholipid complexes (phytosomes): A novel strategy to improve the bioavailability of active constituents. Asian J Pharm Sci. 2019;14(3):265-274. doi:10.1016/ j.ajps.2018.05.011

22. Kuche K, Bhargavi N, Dora CP, Jain S. Drug-phospholipid complex-a go through strategy for enhanced oral bioavailability. AAPS Pharm Sci Tech. 2019;20(2):43. doi:10.1208/s12249-0181252-4

23. Dinda B, Dinda S, DasSharma S, Banik R, Chakraborty A, Dinda M. Therapeutic potentials of baicalin and its aglycone, baicalein against inflammatory disorders. Eur J Med Chem. 2017;131:68-80. doi:10.1016/j.ejmech.2017.03.004

24. Kimura Y, Sumiyoshi M. Effects of baicalein and wogonin isolated from Scutellaria baicalensis roots on skin damage in acute UVB-irradiated hairless mice. Eur J Pharmacol. 2011;661 (1):124-132. doi:10.1016/j.ejphar.2011.04.033

25. Dey S, Ganguly D, Mazumder R, Majumdar S. Enhanced topical permeability of natural flavonoid baicalein through nano liposomal gel: in vitro and in vivo investigation. J Drug Deliv Sci Technol. 2020;101666.

26. Meng L, Xia X, Yang Y, et al. Co-encapsulation of paclitaxel and baicalein in nanoemulsions to overcome multidrug resistance via oxidative stress augmentation and P-glycoprotein inhibition. Int J Pharm. 2016;513(1):8-16. doi:10.1016/j.ijpharm.2016.09.001

27. Liao H, Gao Y, Lian C, et al. Oral absorption and lymphatic transport of baicalein following drug-phospholipid complex incorporation in self-microemulsifying drug delivery systems. Int $J$ Nanomed. 2019;14:7291-7306. doi:10.2147/IJN.S214883

28. Zhou Y, Dong W, Ye J, et al. A novel matrix dispersion based on phospholipid complex for improving oral bioavailability of baicalein: preparation, in vitro and in vivo evaluations. Drug Deliv. 2017;24 (1):720-728. doi:10.1080/10717544.2017.1311968

29. Liu L, Zhou C, Xia X, Liu Y. Self-assembled lecithin/chitosan nanoparticles for oral insulin delivery: preparation and functional evaluation. Int J Nanomed. 2016;11:761-769. doi:10.2147/IJN.S96146

30. Chen S, Han Y, Jian L, Liao W, Zhang Y, Gao Y. Fabrication, characterization, physicochemical stability of zein-chitosan nanocomplex for co-encapsulating curcumin and resveratrol. Carbohydr Polym. 2020;236:116090. doi:10.1016/j.carbpol.2020.116090

31. Qi X, Dong Y, Wang H, Wang C, Li F. Application of Turbiscan in the homoaggregation and heteroaggregation of copper nanoparticles. Colloid Surf A-Physicochem Eng Asp. 2017;535:96-104. doi:10.1016/j.colsurfa.2017.09.015

32. Gagliardi A, Bonacci S, Paolino D, et al. Paclitaxel-loaded sodium deoxycholate-stabilized zein nanoparticles: characterization and in vitro cytotoxicity. Heliyon. 2019;5(9):e02422. doi:10.1016/j.heliyon.2019.e02422

33. Marianecci C, Paolino D, Celia C, Fresta M, Carafa M, Alhaique F. Non-ionic surfactant vesicles in pulmonary glucocorticoid delivery: characterization and interaction with human lung fibroblasts. J Control Release. 2010;147(1):127-135. doi:10.1016/j.jconrel. 2010.06.022

34. He L, Li S, Chung CTW, Gao C, Xin JH. Constructing safe and durable antibacterial textile surfaces using a robust graft-to strategy via covalent bond formation. Sci Rep. 2016;6:36327. doi:10.1038/ srep36327

35. Lee H-S, Kim M-R, Park Y, et al. Fermenting red ginseng enhances its safety and efficacy as a novel skin care anti-aging ingredient: in vitro and animal study. $J$ Med Food. 2012;15(11):1015-1023. doi:10.1089/jmf.2012.2187 
36. Ilk S, Saglam N, Ozgen M. Kaempferol loaded lecithin/chitosan nanoparticles: preparation, characterization, and their potential applications as a sustainable antifungal agent. Artif Cells Nanomed Biotechnol. 2017;45(5):907-916. doi:10.1080/21691401.2016.119 2040

37. Luesakul U, Puthong S, Sansanaphongpricha K, Muangsin N. Quaternized chitosan-coated nanoemulsions: A novel platform for improving the stability, anti-inflammatory, anti-cancer and transdermal properties of Plai extract. Carbohydr Polym. 2020;230:115625. doi:10.1016/j.carbpol.2019.115625

38. Barenholz Y, Amselem S, Goren D, et al. Stability of liposomal doxorubicin formulations: problems and prospects. Med Res Rev. 1993;13(4):449-491. doi:10.1002/med.2610130404

39. Heurtault B, Saulnier P, Pech B, Proust J-E, Benoit J-P. Physicochemical stability of colloidal lipid particles. Biomaterials. 2003;24 (23):4283-4300. doi:10.1016/S0142-9612(03)00331-4

40. Sun Y, Deac A, Zhang GGZ. Assessing physical stability of colloidal dispersions using a turbiscan optical analyzer. Mol Pharm. 2019;16 (2):877-885. doi:10.1021/acs.molpharmaceut.8b01194

41. Celia C, Trapasso E, Cosco D, Paolino D, Fresta M. Turbiscan Lab ${ }^{\mathbb{R}}$ Expert analysis of the stability of ethosomes ${ }^{\circledR}$ and ultradeformable liposomes containing a bilayer fluidizing agent. Colloid Surf B-Biointerfaces. 2009;72(1):155-160. doi:10.1016/j.colsurfb.2009.03.007

42. Liu B, Zhu Y, Tian J, et al. Inhibition of oil digestion in pickering emulsions stabilized by oxidized cellulose nanofibrils for low-calorie food design. RSC Adv. 2019;9(26):14966-14973. doi:10.1039/ C9RA02417D

43. Chhonker YS, Prasad YD, Chandasana H, et al. Amphotericin-B entrapped lecithin/chitosan nanoparticles for prolonged ocular application. Int J Biol Macromol. 2015;72:1451-1458. doi:10.1016/ j.ijbiomac.2014.10.014
44. Nainwal N, Jawla S, Singh R, Saharan VA. Transdermal applications of ethosomes - a detailed review. J Liposome Res. 2019;29 (2):103-113. doi:10.1080/08982104.2018.1517160

45. Celia C, Cilurzo F, Trapasso E, Cosco D, Fresta M, Paolino D. Ethosomes $^{\circledR}$ and transfersomes ${ }^{\circledR}$ containing linoleic acid: physicochemical and technological features of topical drug delivery carriers for the potential treatment of melasma disorders. Biomed Microdevices. 2012;14 (1):119-130. doi:10.1007/s10544-011-9590-y

46. Cristiano MC, Froiio F, Spaccapelo R, et al. Sulforaphane-loaded ultradeformable vesicles as a potential natural nanomedicine for the treatment of skin cancer diseases. Pharmaceutics. 2020;12(1):6. doi:10.3390/pharmaceutics12010006

47. Paolino D, Celia C, Trapasso E, Cilurzo F, Fresta M. Paclitaxelloaded ethosomes ${ }^{\circledR}$ : potential treatment of squamous cell carcinoma, a malignant transformation of actinic keratoses. Eur J Pharm Biopharm. 2012;81(1):102-112. doi:10.1016/j.ejpb.2012.02.008

48. Paolino D, Cosco D, Cilurzo F, et al. Improved in vitro and in vivo collagen biosynthesis by asiaticoside-loaded ultradeformable vesicles. J Control Release. 2012;162(1):143-151. doi:10.1016/j. jconrel.2012.05.050

49. Tan Q, Liu W, Guo C, Zhai G. Preparation and evaluation of quercetin-loaded lecithin-chitosan nanoparticles for topical delivery. Int J Nanomed. 2011;6:1621-1630. doi:10.2147/IJN.S22411

50. Nan W, Ding L, Chen H, et al. Topical use of quercetin-loaded chitosan nanoparticles against Ultraviolet B radiation. Front Pharmacol. 2018;9:826.

51. Zhai Y, Xu R, Wang Y, Liu J, Wang Z, Zhai G. Ethosomes for skin delivery of ropivacaine: preparation, characterization and ex vivo penetration properties. J Liposome Res. 2015;25(4):316-324. doi:10.3109/08982104.2014.999686
International Journal of Nanomedicine

\section{Publish your work in this journal}

The International Journal of Nanomedicine is an international, peerreviewed journal focusing on the application of nanotechnology in diagnostics, therapeutics, and drug delivery systems throughout the biomedical field. This journal is indexed on PubMed Central, MedLine, CAS, SciSearch ${ }^{\circledR}$, Current Contents ${ }^{\circledR} /$ Clinical Medicine,

\section{Dovepress}

Journal Citation Reports/Science Edition, EMBase, Scopus and the Elsevier Bibliographic databases. The manuscript management system is completely online and includes a very quick and fair peer-review system, which is all easy to use. Visit http://www.dovepress.com/ testimonials.php to read real quotes from published authors. 